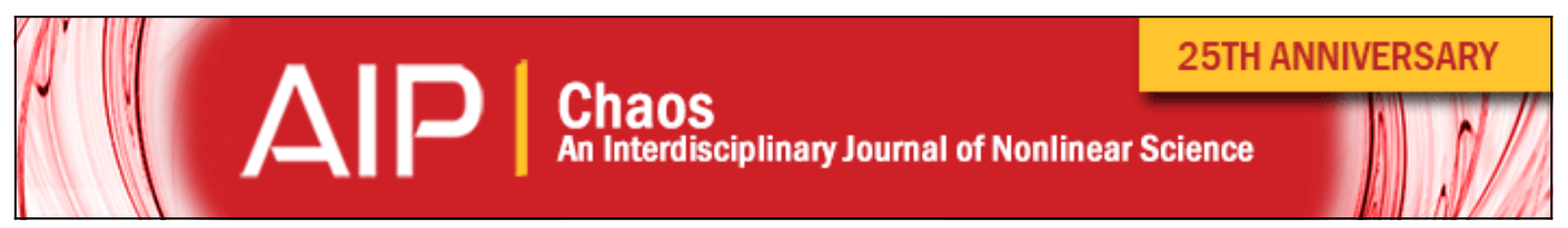

\title{
A complex systems analysis of stick-slip dynamics of a laboratory fault
}

David M. Walker, Antoinette Tordesillas, Michael Small, Robert P. Behringer, and Chi K. Tse

Citation: Chaos 24, 013132 (2014); doi: 10.1063/1.4868275

View online: http://dx.doi.org/10.1063/1.4868275

View Table of Contents: http://scitation.aip.org/content/aip/journal/chaos/24/1?ver=pdfcov

Published by the AIP Publishing

\section{Articles you may be interested in}

Stick-slip instabilities in the shear flow of magnetorheological suspensions

J. Rheol. 57, 1101 (2013); 10.1122/1.4804358

Measuring creep and stick-slip behavior in 2-dimensional photoelastic granular medium

AIP Conf. Proc. 1542, 469 (2013); 10.1063/1.4811969

Stick-slip dynamics of an oscillated sessile drop

Phys. Fluids 21, 072104 (2009); 10.1063/1.3174446

Continuous Nonlinear Delayed-Feedback Dynamics from Noisy Observations

AIP Conf. Proc. 622, 163 (2002); 10.1063/1.1487531

Complexity of EEG-signal in Time Domain - Possible Biomedical Application

AIP Conf. Proc. 622, 155 (2002); 10.1063/1.1487530

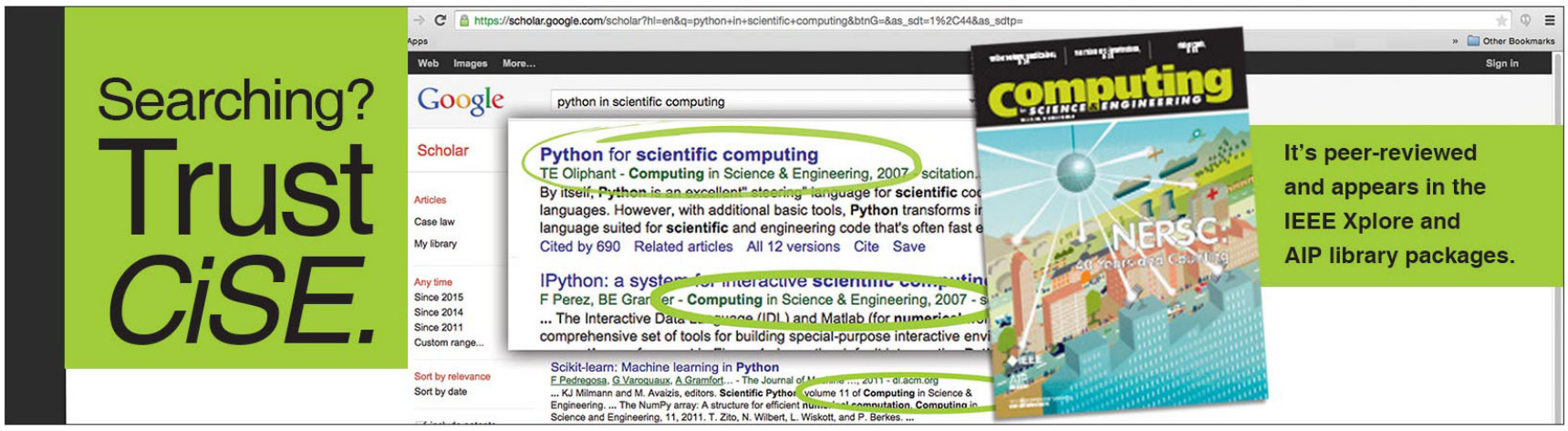




\title{
A complex systems analysis of stick-slip dynamics of a laboratory fault
}

\author{
David M. Walker, ${ }^{1}$ Antoinette Tordesillas, ${ }^{1, a)}$ Michael Small, ${ }^{2}$ Robert P. Behringer, ${ }^{3}$ \\ and Chi K. Tse ${ }^{4}$ \\ ${ }^{1}$ Department of Mathematics and Statistics, University of Melbourne, Parkville VIC 3010 Australia \\ ${ }^{2}$ School of Mathematics and Statistics, University of Western Australia, Crawley WA 6009, Australia \\ ${ }^{3}$ Department of Physics, Duke University, Durham, North Carolina 27708, USA \\ ${ }^{4}$ Department of Electronic and Information Engineering, Hong Kong Polytechnic University, Hung Hom, \\ Kowloon, Hong Kong
}

(Received 18 June 2013; accepted 1 March 2014; published online 13 March 2014)

\begin{abstract}
We study the stick-slip behavior of a granular bed of photoelastic disks sheared by a rough slider pulled along the surface. Time series of a proxy for granular friction are examined using complex systems methods to characterize the observed stick-slip dynamics of this laboratory fault. Nonlinear surrogate time series methods show that the stick-slip behavior appears more complex than a periodic dynamics description. Phase space embedding methods show that the dynamics can be locally captured within a four to six dimensional subspace. These slider time series also provide an experimental test for recent complex network methods. Phase space networks, constructed by connecting nearby phase space points, proved useful in capturing the key features of the dynamics. In particular, network communities could be associated to slip events and the ranking of small network subgraphs exhibited a heretofore unreported ordering. (c) 2014 AIP Publishing LLC. [http://dx.doi.org/10.1063/1.4868275]
\end{abstract}

Inferring dynamical structure of a deterministic nonlinear system from bulk time series measurements contaminated by noise remains a challenge, despite a number of available and complementary methods for characterization. Here, we demonstrate a variety of complex system methods to a problem of particular interest to geophysics, i.e., granular friction. Knowledge of granular friction is crucial for understanding not only the mechanisms responsible for earthquake dynamics but also the details of force transmission in granular-structure/solid interaction systems (e.g., soil-vehicle interaction, building foundations, etc.). We study a slider experiment introduced to investigate such behavior. Our analysis indicates that the dynamics of granular friction within the slider are consistent with short term determinism, possibly quasiperiodic, but with no long-term memory. The observed dynamics also induce a complex network representation possessing a previously unobserved prevalence ranking of small subgraphs, i.e., a new superfamily. Our characterization suggests that despite models based on periodicity being sufficient for some purposes, new models are needed to fully capture the complex dynamics responsible for the observed slider behavior.

\section{INTRODUCTION}

When two frictional solid objects move relative to each other, the motion of their surfaces often alternate between periods of stick contact, when surfaces are locked together, and periods of sliding contact, when surfaces overcome "friction" and slip past each other. This phenomenon, known

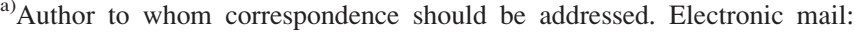
atordesi@unimelb.edu.au
}

commonly as stick-slip, arises in many aspects of everyday life and is often implicated in damage and failure of materials. Stick-slip is by no means limited to the dry contact of two solid bodies: other examples include articular cartilage damage, ${ }^{1}$ vibrations in vehicle suspensions and brake systems, ${ }^{2,3}$ erratic motion in industrial machinery and tools, ${ }^{4}$ and the behavior of active geological faults. ${ }^{5-7}$ Indeed, stickslip motion can arise in the presence of fluids between surfaces $^{8}$ and in discrete granular materials. ${ }^{9-14}$ Regardless of the materials involved, what makes stick-slip one of the most challenging dynamical phenomenon to characterize and model is that it is the result of an interaction between many complex entwined mechanical (and sometimes chemical) processes. ${ }^{1-17}$

Our interest here lies in the occurrence of stick-slip in granular materials, which often manifest in fluctuations of macroscopic shear stresses-a measure of the strength of a material. For these highly ubiquitous and technologically important class of materials, understanding stick-slip is not only of core importance to the study of granular friction but of a number of related processes, including: frictional strength of geological fault gouge, shear bands, the coexistence of stable solid-like and flowing liquid-like regimes, and the existence and uniqueness of a so-called "critical state" of a material (e.g., Refs. 9-16, 18, and 19). In many practical settings, arguably the most valuable piece of information embodied in stick-slip is the dynamics of energy storage and dissipation in the system. Understanding the energetics and recurrence of earthquakes, and the optimal control of energy consumption in many particulate processes (e.g., comminution, dewatering, etc.), hinges on our ability to unravel the micromechanical details underlying stick versus slip for a broad gamut of loading conditions. ${ }^{18,20-23}$ 
Extant studies of granular materials under shear and compression have uncovered a connection between stick-slip and the dual nature of force transmission in granular systems (e.g., Refs. 11, 16, and 22-25). In particular, studies have shown that stick-slip dynamics is governed by the continual evolution of so-called force chains, i.e., self-organized columnar structures comprising particles, which carry the majority of the load in the system. These structures, belonging to the so-called strong network ${ }^{26}$ - the subset of inter-particle contacts that carry above average contact force relative to all contactscontinually align in the direction of the most compressive principal stress, and are thus axially loaded. Surrounding the force chains are the particles in the complementary weak network, the subset of contacts not in the strong network, which serve to provide chains the necessary lateral support. Using photoelastic disk assemblies, experiments ${ }^{9,24,25,27-31}$ directly revealed the tie between the creation and collapse of force chains, and the rises and falls in the macroscopic shear stress. Observations of these stress fluctuations strongly suggest that stick events are governed by the formation of force chains, while slip is due to the collective failure of force chains by buckling and elastic unloading: see Fig. 1. During stick, stored elastic strain energy builds up at the contacts between the constituent particles of the force chain. This build up of stored energy during stick cannot, however, continue indefinitely. Force chains are highly unstable structures and, like architectural columns, are prone to fail by buckling when overloaded to some critical value. Thus, when a critical threshold is reached, force chains buckle and the stored energy accumulated at their constituent contacts is collectively released. Studies of granular drag ${ }^{11-13}$ and granular shearing ${ }^{22,23}$ have also shed considerable light on the possible role of force chains and, in particular, the influences of particle surface and shape (e.g., brass, wood, pasta) on force chain stability and, in turn, on the dynamical features of the observed fluctuation (i.e., random versus periodic). More recent studies on photoelastic disk experiments, ${ }^{31-33}$ supplemented by computer simulations $^{34-39}$ further characterize the topology and dynamics of force networks.

In this study, we analyze time series of a proxy for granular friction for a set of slider experiments. Our objective is to characterize the dynamical structure of stick-slip, specifically: the number of irreducible degrees of freedom of the system, the nature of the dynamics (periodic, chaotic, etc.), including partitions of phase space as identified from community structures of networks constructed from the time series data. We note that connecting the macroscopic behavior of this study to the earlier discussion on the mesoscopic analysis of force chain evolution is outside the scope of this paper. However, our future goal is to make this connection, hence points of commonality (e.g., what network communities tell us about the dynamics of stick-slip at the macro and mesoscales) are of interest.

The remainder of this paper is organized as follows. In Sec. II, we describe the slider experiment and the resulting macroscopic time series. In Sec. III, we characterize the dynamics of the time series via a suite of techniques from nonlinear time series analysis. In Sec. IV, we transform the time series to a complex network and characterize these networks using a set of network statistics: different aspects of the stick-slip dynamics are associated with various topological structures and properties of the networks. Finally, we summarize our results in Sec. V.

\section{EXPERIMENT AND DATA SETS}

A slider of mass $M$ is pulled over a quasi-two-dimensional granular surface that consists of a vertically oriented layer of photoelastic disks. A small cart moves horizontally on a track above the layer. Mounted on the cart is a force gauge; a spring of force constant $k_{s}$ connects the force gauge to the front of the slider. The cart is driven by a stepper motor and lead screw at a constant velocity, $v$. Note that if the slider is static, as it is, essentially, during stick events, then $F_{p}+F_{g}=0$, where $F_{g}$ and $F_{p}$ are respectively the force on the slider from the grains, and the force on the slider by the pulling apparatus. Thus, $F_{p}=-F_{g}$ during stick. Since the cart always moves at a fixed speed, the signature of a stick event is an $F_{p}$ that increases linearly in time. The slope of such an increasing curve $F_{p}(t)$ is roughly $d F / d t \simeq v k_{s}$, where in fact the slope is somewhat smaller than $v k_{s}$ due to the finite elasticity of the static granular material. During slip events, when the slider may be accelerating, $F_{p}=M a-F_{g}$. At the beginning of a slip event, the cart acceleration, $a$, is often large and positive; hence, a slip event is typically signalled by a precipitous drop in $F_{p}$. A typical stick-slip cycle consists of a steadily increasing $F_{p}$, followed by a rapid drop of $F_{p}$.

Our granular system consists of photoelastic disks (PSM, Vishay Measurements, elastic modulus $\sim 4 \mathrm{MPa}$, Poisson ratio

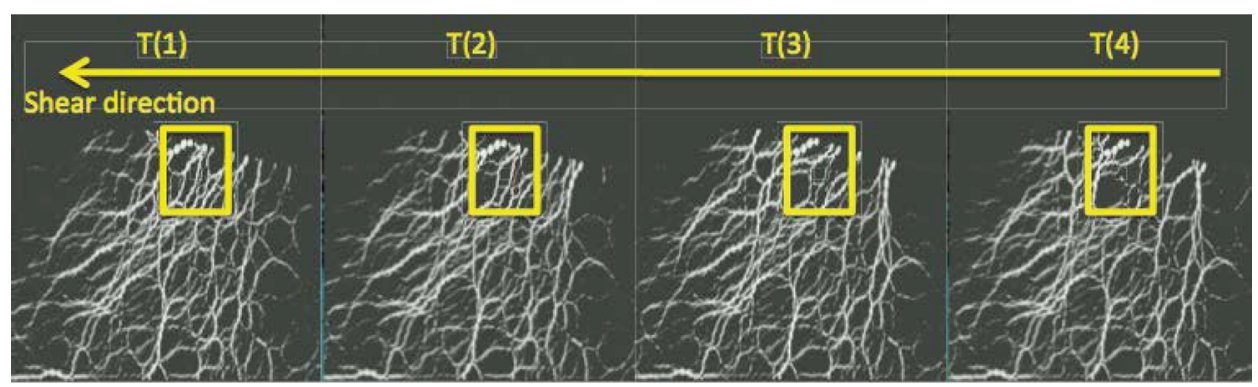

$\mathrm{T}(1)<\mathrm{T}(2)<\mathrm{T}(3)<\mathrm{T}(4)$

FIG. 1. Underlying evolution of load-bearing force chains during stick and slip. Force chains form and accumulate stored energy at the contacts during a stick event. Force chains collapse by buckling, with all of the stored energy that was accumulated at the contacts from the preceding stick event released collectively during the slip event. Boxed areas highlight the failure of a force chain by buckling as shearing progresses over time $T(1)<T(2)<T(3)<T(4)$. 
$\sim 0.4$ ) that are placed in a channel between two vertically oriented Plexiglas sheets. This allows simultaneous measurement of $F_{p}$, motion of the slider, and the particle-scale response within the granular layer. The channel is $1.5 \mathrm{~m}$ long, $15.5 \mathrm{~cm}$ tall, and has a gap slightly greater than the thickness of the disks, which is $3.2 \mathrm{~mm}$. The depth of the granular layer corresponds to $\sim 20$ mean grain diameters. The disks are bidisperse, with diameters $4 \mathrm{~mm}$ and $5 \mathrm{~mm}$, and thickness $3.2 \mathrm{~mm}$. The average particle size is $d=4.37 \mathrm{~mm}$. The sliders are cut from either brass or aluminum and are roughened on the bottom surface (which contacts the grains) by a series of half-round cut-outs, of diameter $d$. The sliders have lengths of $30 d$ to $60 d$, and masses between $50 \mathrm{~g}$ and $250 \mathrm{~g}$.

The force gauge cart also carries an imaging system for determining the photoelastic response of the grains. This consists of a circularly polarized light source that moves along one side of the channel and a second crossed circular polarizer that is placed in front of a high speed video camera that operates at frame rates from $30 \mathrm{~Hz}$ to $500 \mathrm{~Hz} . F_{p}$ data are acquired synchronously with camera frame grabs. Stressed photoelastic materials placed between crossed polarizers show light and dark bands which provide quantitative information on inter-particle forces ${ }^{27,28,31}$ (see images in Fig. 1). In this work, however, we concentrate solely on an analysis of the time series data, leaving the coupled analysis of images and time series to a future exploration.

A number of time series were recorded with different experimental test parameters, in particular, duration of experiment, sampling frequency, cart mass, and cart speed. We report the results of a combined nonlinear time series and complex network analysis for a number of these runs to see if there is consistency across each run, and attempt to characterize the underlying properties of a dynamical system capable of explaining the observed data.

In Table I, we provide details of the duration, sampling time, and speed of the cart. Each run resulted in a different number of data points. To check for consistency in observed dynamics across tests, however, we applied the following down-sampling procedure. Using the cart speed $V$ and time of experiment, we calculated the expected travel distance of the cart. Of course, due to the very essence of the stick-slip behavior, the cart will not have traveled this distance exactly. We then selected a consistent (expected) travel distance for all tests, and sampled the data so that each time series contained 21000 points across this distance. After following this procedure, we noted that one of the tests (TS10) had too much observational noise corruption, and we dropped this test from our analysis and reporting. In Figs. 2(a) and 2(b), we present two of the slider time series (TS7 and TS13). A calculation of the Fourier Power spectrum did not show any particularly dominant peak. This lack of strong evidence of periodicity suggests there are more complex, perhaps deterministic, dynamics present in the underlying mechanism responsible for the observed stick-slip behavior. Previously, similar data from the same experimental apparatus has demonstrated that the statistics of the stick-slip events follow scaling laws similar to Gutenberg-Richter power laws. ${ }^{40}$
TABLE I. Experimental measurement parameters and basic dynamical data analysis parameters. Note TS10 contained a significant level of chatter in its measurements so we do not report the results here. All time series were down-sampled to have the same number of recordings for the same cart travel distance (i.e., $v \times$ Time). $T, d_{E}$, and $d_{L}$ refer to time-delay lag, global embedding dimension, and local dimension, respectively, The checkmarks and crosses for PPS and ATS indicate evidence for rejecting $(\checkmark)$ and failure to reject $(\times)$ the null hypothesis, respectively. Italics indicate the slider time series selected in figures to display the results of various calculations.

\begin{tabular}{lcccccccc}
\hline \hline Data & Time (s) & Freq. (Hz) & $v(\mathrm{~cm} / \mathrm{s})$ & $T$ & $d_{E}$ & $d_{L}$ & PPS & ATS \\
\hline TS1 & 3600 & 120 & 0.33 & 20 & 8 & 6 & $\times$ & $\times$ \\
TS2 & 3600 & 120 & 0.33 & 20 & 10 & 5 & $\checkmark$ & $\times$ \\
TS3 & 3600 & 120 & 0.33 & 20 & 12 & 5 & $\checkmark$ & $\times$ \\
TS4 & 1800 & 240 & 0.66 & 20 & 9 & 5 & $\times$ & $\times$ \\
TS5 & 900 & 480 & 0.13 & 20 & 9 & 5 & $\times$ & $\times$ \\
TS6 & 450 & 960 & 0.26 & 20 & 8 & 5 & $\checkmark$ & $\times$ \\
TS7 & 28 & 1000 & 4.22 & 20 & 6 & 4 & $\times$ & $\times$ \\
TS8 & 225 & 1920 & 0.53 & 20 & 9 & 4 & $\times$ & $\times$ \\
TS9 & 113 & 3823 & 1.05 & 20 & 6 & 4 & $\times$ & $\times$ \\
TS10 & 28 & 4000 & 4.22 & $\ldots$ & $\ldots$ & $\ldots$ & $\ldots$ & $\ldots$ \\
TS11 & 28 & 7714 & 4.22 & 20 & 5 & 4 & $\times$ & $\times$ \\
TS12 & 56 & 7714 & 2.11 & 20 & 5 & 4 & $\times$ & $\times$ \\
TS13 & 28 & 15429 & 4.22 & 20 & 5 & 4 & $\checkmark$ & $\times$ \\
\hline \hline
\end{tabular}
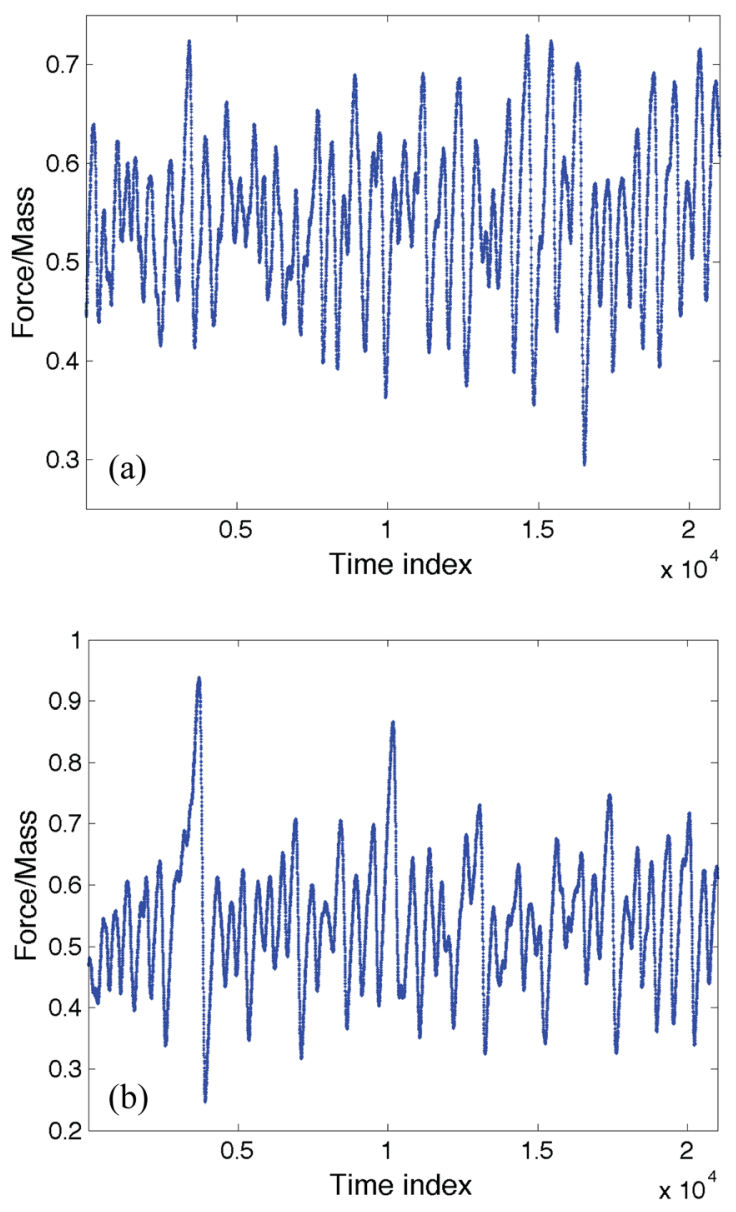

FIG. 2. Two examples of the slider time series (a) TS7 and (b) TS13. 


\section{NONLINEAR TIME SERIES METHODS}

We apply tools from nonlinear time series analysis to gain more knowledge about the slider system and, in turn, stick-slip dynamics. It is important to first: (i) check whether the observed data is sufficiently stationary for nonlinear methods to be usefully applied, and (ii) determine whether a linear approach does not already tell us everything of practical interest about the data. A definitive check of stationarity of a finite length data set is impossible. However, a basic test is to determine whether the fluctuations of the sample mean over contiguous segments of the data are within the fluctuations observed in the data of each segment. ${ }^{41}$ By segmenting each of the observed slider time series into contiguous data sets, each of length $n$, we found the fluctuations of the running sample mean for $n>500$ was within the sample standard deviation. Thus, over segments of length $n>500$, the slider data can be considered approximately stationary.

\section{A. Linear surrogates}

We test whether or not the observed data is fully explained by simple linear descriptions using the method of surrogate data. Surrogate data testing is a form of randomization testing. Additional data sets are generated which are consistent with the observed data set and consistent with a stated hypothesis. ${ }^{42,43}$ A hypothesis is tested by calculating a test statistic and comparing the value obtained from the original data to the distribution of test statistic values from the surrogates. We use z-scores to measure the deviation of the original data test value from the sample mean of the surrogate values in terms of the surrogates sample standard deviation. If the z-score is high enough, we can reject the null hypothesis under consideration. Z-scores rely on a Gaussian assumption and so other metrics such as the KolmogorovSmirnov test may be more reliable and appropriate. ${ }^{42}$

The three standard surrogate tests that we use are coined Algorithm 0, 1, and 2. In Alg. 0, the data set is tested against the hypothesis that it is independently and identically distributed noise. This involves generating uncorrelated data with the same probability distribution of the original data. Algorithm 1 tests the hypothesis that the data set is linearly filtered noise; hence, random surrogates with the same power spectrum and autocorrelation function are generated. Algorithm 2 tests the hypothesis that the data are a monotonic nonlinear transformation of linearly filtered noise. The hypotheses of Alg. 0 is a subset of Alg. 1, which is itself a subset of Alg. 2. However, the realizations obtained from each algorithm do not necessarily preserve this nesting (see, e.g., Small ${ }^{42}$ for a description of surrogate generation methods for each algorithm).

Here, we reject null hypotheses for a z-score above 2; however, most runs and surrogate tests return z-score values above 3 . We used the average mutual information (AMI) ${ }^{44,45}$ at lag one as the test statistic. The average mutual information is a nonlinear measure over a range of lags. However, as in other studies, ${ }^{46}$ we found checking only lag one provided sufficient evidence to reject the null hypothesis for each test. We found we could reject each hypothesis, hence a simple linear description of the observed slider time series does not fully explain the observed behavior.

\section{B. Reconstructed state space}

The analysis of time series using the methods of nonlinear dynamics is predominantly based around studying the data in a reconstructed phase space. ${ }^{45}$ A common method of finding such a phase space is time-delay embedding, where the time series $\left\{y_{t}\right\}_{t=1}^{N}$ and delayed versions of itself are used to form the coordinates. ${ }^{47}$ The state $z \in R^{d_{E}}$ of the reconstructed phase space is

$$
z_{t}=\left(y_{t-\left(d_{E}-1\right) T}, \ldots, y_{t-T}, y_{t}\right),
$$

where the parameters of this reconstruction are the timedelay $(T)$ and the embedding dimension $\left(d_{E}\right)$. There are prescriptions for selecting these parameters. The first minimum of the AMI determines the time delay lag. AMI can be considered as a kind of nonlinear autocorrelation function ${ }^{44}$ and selecting $T$ as the first minimum helps to identify more independent coordinates in the reconstructed space. For a given $T$, the value of $d_{E}$ can be selected using the method of false nearest neighbours ${ }^{45}$ (FNN). FNN attempts to find the smallest number of coordinates required to unfold the observed data and minimize self-crossing of trajectories. That is, coordinates in phase space are close because of the dynamics and not because of an insufficient projection to a lower dimensional space.

We found the first minimum of average mutual information at $T=20$, although a clearer minimum occurred for higher values of $T$ in some tests. For the majority of the tests, however, $T=20$ was closer to a quarter of the length of a typical stick-slip event. Fixing $T=20$, we found a suitable global embedding dimension $d_{E}$ from false nearest neighbors to lie in the range 5-12 (see, Table I). However, $d_{E}=8$ appeared to be adequate for all tests. Thus, for all future calculations, the parameters of the reconstructed phase space are $T=20$ and $d_{E}=8$.

The global embedding dimension $d_{E}$ is based solely on geometric arguments. Consequently, it will likely return a value greater than the actual dimension of the system. It is possible to determine the number of active degrees of freedom of the system using the method of local false nearest neighbors ${ }^{48}$ (LFNN). LFNN adds to the geometric considerations of FNN by including the predictability of the dynamical information inherent in the time series. The local dimension $d_{L}$ provides the number of variables required to accurately model the evolution of local neighborhoods of phase space. We can find the number of these variables by examining $P_{x}$ - the ratio of the number of points poorly predicted by a local model over $T=20$ time steps to the number of pointsfor different-sized local neighborhoods across dimensions. In this context, a point is poorly predicted if the $L^{1}$ prediction error is larger than $10 \%$ of the spread of the data. These dynamical variables are the ones which are active for a dynamical description of the physics. They differ from a more standard interpretation of degrees of freedom, which would not only include these active variables but also those 
variables deemed inactive with respect to the dynamics due to, for example, damping.

In Fig. 3 and Table I, we see $P_{x}$ falls to a floor for $d_{L}=4-6$, i.e., the slider exhibits low dimensional dynamics with four to six dynamical degrees of freedom. Thus, models capable of capturing the macroscopic stick-slip dynamics of granular friction should have no fewer than fourto-six dynamical variables.

\section{Attractor trajectory surrogates (ATS)}

Given a reconstructed state space, we can pose more specific questions about the observed dynamics using pseudoperiodic surrogates (PPS) or ATS, depending on the specific null hypothesis. ${ }^{42,49}$ For oscillatory time series, like the sequential stick-slip events in the slider (Fig. 2), PPS test for periodic dynamics driven by uncorrelated noise. ATS test whether pseudo-cycle (stick-slip events) dynamics is preserved with no evidence for long term dynamical correlations between cycles (cf. the cycle-shuffle surrogates in Ref. 50). For the slider, PPS are effectively testing whether the slider dynamics within stick-slip events is consistent with noisedriven periodic dynamics or, more complicated nonlinear determinism. In contrast, ATS test whether the dynamics of stick-slip events is the result of the same underlying dynamics with no long term correlations between the different events: that is, whether the granular bed only has short term memory.

PPS and ATS are a local phase space resampling of the original time series with replacement based on the resolution of resampling. A prescription for determining a resolution is based on how many contiguous segments of the original time series, each of non-trivial length, appear within the surrogate. The length of contiguous segments determines the level of randomization in the algorithm. The length of contiguous segments, in turn, is determined by the level of randomization-or, equivalently, the neighborhood size. With a large neighborhood, sampling with replacement is essentially random and irrespective of the phase space structure: this is equivalent to Alg. 0 surrogates-random resampling (albeit with replacement). At the other end of the scale, with a very small neighborhood, there is minimal

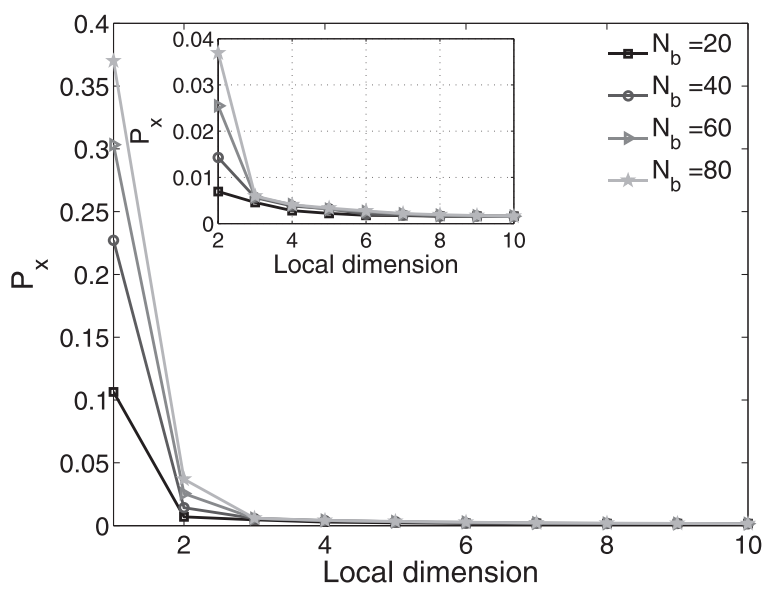

FIG. 3. TS13: local false nearest neighbors for different number of near neighbors $\left(N_{B}\right)$. Inset: zoomed in showing $d_{L}=4-6$ local dimensions. A time-delay of $T=20$ was used to embed the time series data. randomization and long segments of the dynamics are reproduced. With a non-trivial level of randomization, this yields ATS. In the trivial case (no noise), one just recovers the original time series. As the noise level (neighborhood size) increases, the original time series is segmented and the segments are shuffled at locations where many similar states occur close to one another in phase space. This is what is required for the ATS algorithm. Increasing noise level (neighborhood size) has the effect of increasing dynamical noise contamination in the surrogate generation process until only the most basic macroscopic dynamical features are retained. That is, the pseudo-periodic structure and hence the PPS algorithm.

We use the nonlinear test statistic of algorithmic complexity to distinguish the observed data from PPS and ATS data (see, e.g., Small ${ }^{42}$ for ways to calculate this complexity measure). In Table I, a " $\checkmark$ " indicates that there is sufficient evidence to reject the null hypothesis (i.e., z-scores above 2) and a " $x$ " indicates failure to reject. We find mixed results for the PPS hypothesis: periodic dynamics plus uncorrelated noise often appears sufficient to describe the observed dynamics. In all cases, we fail to reject the ATS null hypothesis. For the length of data, ATS are unable to detect long term dynamical correlations between stick-slip events with respect to the test statistic of algorithmic complexity. This can be explained by the physical fact that disturbed and undisturbed sets of grains come into play as the slider moves unidirectionally along the granular bed.

We can view these findings side-by-side with results from an earlier study on the macroscopic stress response behavior of dense granular media undergoing deformation in the presence of a fully developed shear band. ${ }^{19}$ In that work, a time series of the macroscopic stress ratio in the large strain regime was studied using nonlinear methods. The observed response of this virtual system was "best" described by nonlinear models exhibiting a complicated nonperiodic dynamics, as is suggested here by PPS for some of the sliders. Furthermore, local false nearest neighbors analysis of the virtual system similarly suggested 4-6 active degrees of freedom are needed to describe the dynamics of stick-slip. This is also consistent with Cosserat constitutive relations based on force chain evolution developed for the large strain regime in the presence of a fully developed shear band. $^{51}$

\section{COMPLEX NETWORK METHODS}

In addition to surrogate testing and phase space reconstruction methods, the technique of obtaining a complex network from time series can garner useful information from the data. There are various ways of transforming an observed time series into a complex network. Zhang and Small ${ }^{52}$ transformed oscillatory time series to a network by segmenting the data into pseudo-cycles - the peak-to-peak segments of the time series which would correspond to the stick-slip cycles in the sliders-and calculating the correlation between pair-wise segments. Network nodes correspond to the pseudo-cycles, and links are made if the correlations are above a specified threshold. The visibility graph method of 
Lacasa et al. ${ }^{53}$ introduced another transformation method, whereby each scalar value of the time series corresponds to a network node and the links are drawn according to the relative magnitude of each value. Both methods produce networks whose respective properties help to characterize the underlying processes that may be responsible for the time series. Moreover, neither method requires a reconstructed phase space to be determined.

$\mathrm{Xu}$ et al. $^{54}$ showed how a complex network can be obtained from a phase space reconstructed using a timedelay embedding. Network nodes are the individual phase space points and connectivity is determined by relative closeness of these points. They coined such a network a Phase Space Network (PSN), and this is the type of network we use to study the slider time series. In a similar vein, Marwan et al. ${ }^{55}$ recognized the likeness between a network adjacency matrix and a recurrent plot matrix, and developed a recurrence-based network representation of a time series. A review by Donner et al. ${ }^{56}$ discusses the relationship between phase space networks and recurrence-based networks from a theoretical and practical standpoint.

The formation of a PSN can be seen as a first step in the dimension reduction process ISOMAP, in particular $k$-ISOMAP. ${ }^{57}$ ISOMAP has been used in dynamical systems data analysis to find a lower dimensional manifold where the dynamics evolve. ${ }^{58,59}$ One constructs this manifold by taking a PSN and forming a matrix from the shortest path length distribution between each pair of nodes in a PSN. A multidimensional scaling step is performed on this matrix to form a new set of (lower dimensional) coordinates to study the dynamics. Notable success has been achieved when these coordinates result in a one-dimensional return map. ${ }^{58,59}$ One dimensional return maps are appealing because they are more amenable to examination by global analysis methods such as Ulam's method for approximating the PerronFrobenius transfer operator. ${ }^{60,61}$ The spectral properties of matrices approximating this operator help to identify global structures in the data, including almost invariant sets as well as providing a means to estimate dynamical invariants. ${ }^{62,63}$

More generally, the Perron-Frobenius transfer operator can be approximated directly from a reconstructed phase space using the Ulam-Galerkin method. ${ }^{64,65}$ In this case, the phase space is covered by regions and the dynamical flow from region to region is approximated by a Ulam-Galerkin matrix that can be interpreted as a (weighted) directed graph. Nodes correspond to the regions and the weighted links encode the transport mechanism. In contrast, the PSNs we consider here do not explicitly encode the time evolution as only closeness in phase space determines the connectivity. Furthermore, nodes in a PSN correspond to observed data points and not regions of phase space. A PSN differs from the Ulam-Galerkin approach by being an unweighted, undirected network although there are clear correspondences worthy of exploration.

For example, in the following, we consider a partitioning of the PSNs using modularity-based methods. In Santitissadeekorn and Bollt, ${ }^{66}$ the network arising from the Ulam-Galerkin matrix approximation is also partitioned using a modularity score to reveal basins and basin barriers in phase space. It is also intriguing to consider how each approach would compare with generating partitions, ${ }^{67}$ as well as the symbolic dynamics ${ }^{68,69}$ within a low dimensional manifold identified via ISOMAP.

Here, we construct PSNs using a fixed connection rule where each node connects to its $k$ closest phase space neighbors. We discount connections based on close temporal correlation, akin to applying a Theiler window to de-correlate close neighbors within a correlation integral calculation. ${ }^{41} \mathrm{~A}$ suitable de-correlation interval or size of Theiler window can be gauged from a space-time separation plot, whereby the quantiles of the inter-point distance distribution are calculated for data separated by a given time span. ${ }^{41}$ We find that the quantiles have started to separate from each other for a de-correlation interval of 100 , so nodes representing reconstructed phase space points temporally closer than 100 time steps are barred from connecting to each other. As before, we use the reconstructed phase space embedding parameters $T=20$ and $d_{E}=8$ resulting in networks with 20860 nodes. We fix $k=4$ neighbors to construct each PSN.

The structure of a PSN can be quantified and described by standard network properties such as average degree (the average number of links connected to a node), average clustering coefficient (a measure of the closed triples in a network), degree assortativity (a correlation score of how nodes with the same degree are connected to each other), average shortest path lengths (the minimum number of links connecting two nodes), node closeness centrality (how closely connected a node is to all other nodes in the network) among others; see, e.g., Newman ${ }^{70}$ for more detailed descriptions. In Table II, these global properties are presented for each PSN. We observe consistency across tests. Each network appears disassortative (negative degree assortativity): i.e.,

TABLE II. Global network properties of the PSN constructed from the slider time series using $T=20, d_{E}=8$, and a Theiler, or de-correlation window of 100 . Each state space point (node) is connected to its 4 closest neighbors. N.B. this means that some nodes can have degree greater than 4 since they may be within the 4 closest neighbors to another node but that node may not be within the 4 th closest neighbors of themselves. The clustering coefficient for a node of degree $k_{i}$ being a part of $n_{i}$ triangles is calculated to be $n_{i} /\left(\begin{array}{c}k_{i} \\ 2\end{array}\right)$.

\begin{tabular}{lccccc}
\hline \hline Data & $\begin{array}{c}\text { Number } \\
\text { of network } \\
\text { components }\end{array}$ & $\begin{array}{c}\text { Average } \\
\text { degree }\end{array}$ & $\begin{array}{c}\text { Degree } \\
\text { assortativity }\end{array}$ & $\begin{array}{c}\text { Average } \\
\text { clustering } \\
\text { coefficient }\end{array}$ & $\begin{array}{c}\text { Average } \\
\text { path } \\
\text { length }\end{array}$ \\
\hline TS1 & 1 & 6.9814 & -0.3295 & 0.0349 & 16.3294 \\
TS2 & 2 & 6.9567 & -0.2689 & 0.0510 & 16.2198 \\
TS3 & 1 & 6.9216 & -0.2686 & 0.0873 & 21.8721 \\
TS4 & 1 & 6.9406 & -0.2259 & 0.0478 & 18.0717 \\
TS5 & 1 & 6.9496 & -0.3171 & 0.0415 & 17.6985 \\
TS6 & 1 & 6.8206 & -0.2906 & 0.0381 & 20.3626 \\
TS7 & 1 & 6.6998 & -0.3146 & 0.0400 & 38.8677 \\
TS8 & 1 & 6.7826 & -0.2210 & 0.0495 & 20.3710 \\
TS9 & 1 & 6.7158 & -0.2534 & 0.0266 & 28.4885 \\
TS11 & 1 & 6.6814 & -0.2424 & 0.0361 & 39.3600 \\
TS12 & 1 & 6.5910 & -0.2581 & 0.0407 & 36.8730 \\
TS13 & 1 & 6.7371 & -0.2269 & 0.0541 & 44.5371 \\
\hline \hline
\end{tabular}


nodes of a given degree are typically connected to nodes of different degrees.

Closeness centrality measures how central a node is within a network. Centrality is based on how close the node is topologically to all other nodes. Distance is measured through the inverse of shortest path length. Nodes with high closeness centrality are closer to all other nodes. This measure of centrality suffers from a lack of robustness as the removal or addition of a link can dramatically change its value; however, we find it useful to highlight a feature of the slider PSNs. We use the Mathematica ${ }^{71}$ implementation of the following formula to calculate closeness centrality for a node $i$ with degree $k_{i}$

$$
c c(i)=\frac{k_{i}}{\sum_{j} d_{i j}},
$$

where $d_{i j}$ is the shortest path distance between nodes $i$ and $j$. We can attribute a PSN node's closeness centrality to its phase space location. Nodes with low values of closeness centrality are more remote in phase space. In Fig. 4, we identify the scalar time series with a reconstructed phase space point, and color it based on its node's closeness centrality value. Nodes with lower closeness centrality are more associated with slip events and, typically, with large slip events. By contrast, nodes with higher closeness centrality values are more associated with the slow build up in force-to-mass ratio, indicative of stick events. In other words, in phase space, trajectories of the dynamics during the stick phase slowly evolve through similar areas of phase space, before dramatically separating during the inception and in the course of slip events.

The set of nodes $V$ of a network can be partitioned into disjoint communities $V_{i}$ based on the relative propensity of intra-community and inter-community connections, such that $V=\cup V_{i}$. A measure of how well the partition promotes intra-community over inter-community links is modularity $Q$; for $K$ communities, this is given by ${ }^{66}$

$$
Q=\sum_{i=1}^{K}\left(e_{i i}-a_{i}^{2}\right)
$$

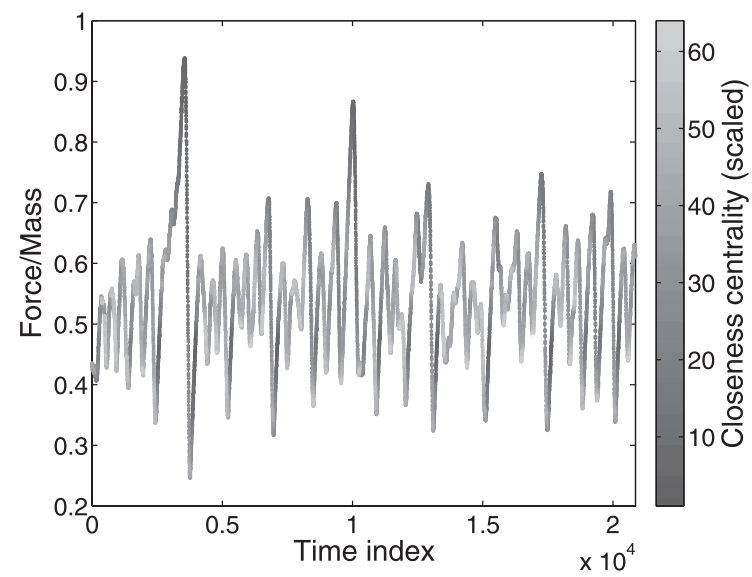

FIG. 4. TS13: Network node closeness centrality values mapped back to the original time series. Values have been scaled to lie in Refs. 1 and 64. where $e_{i i}$ is the fraction of links which have both end-point nodes within community $V_{i}$, and $a_{i}$ is the fraction of links with at least one end-point node in community $V_{i}$. The goal is to find a set of communities $V_{i}$ which maximizes $Q$. We use the Mathematica $^{71}$ implementation, which follows Clauset ${ }^{72}$ by using a greedy algorithm to find an approximate sub-optimal partition. The implementation is initialized by assigning each node to its own community. Pairs of communities are merged and $Q$ is re-evaluated. The pair of communities whose joining produces the greatest increase in $Q$ are merged. The implementation stops when no joining of pairs of communities increases $Q$. This implementation is different to Santitissadeekorn and Bollt, ${ }^{66}$ which allows pairs that lead to the smallest decrease in $Q$ to merge when no pair leads to an increase in $Q$.

The time series can be visualized by coloring its values according to their associated PSN node community membership. Fig. 5(a) shows that the majority of points, and hence the phase space dynamical behavior, are confined to a few communities (similar color indicates the same community assignment). Comparing with Fig. 4 reveals these points also correspond to nodes with high closeness centrality. Local slip events appear to reside in their own communities distinct from the typical behavior. We can quantify this observation
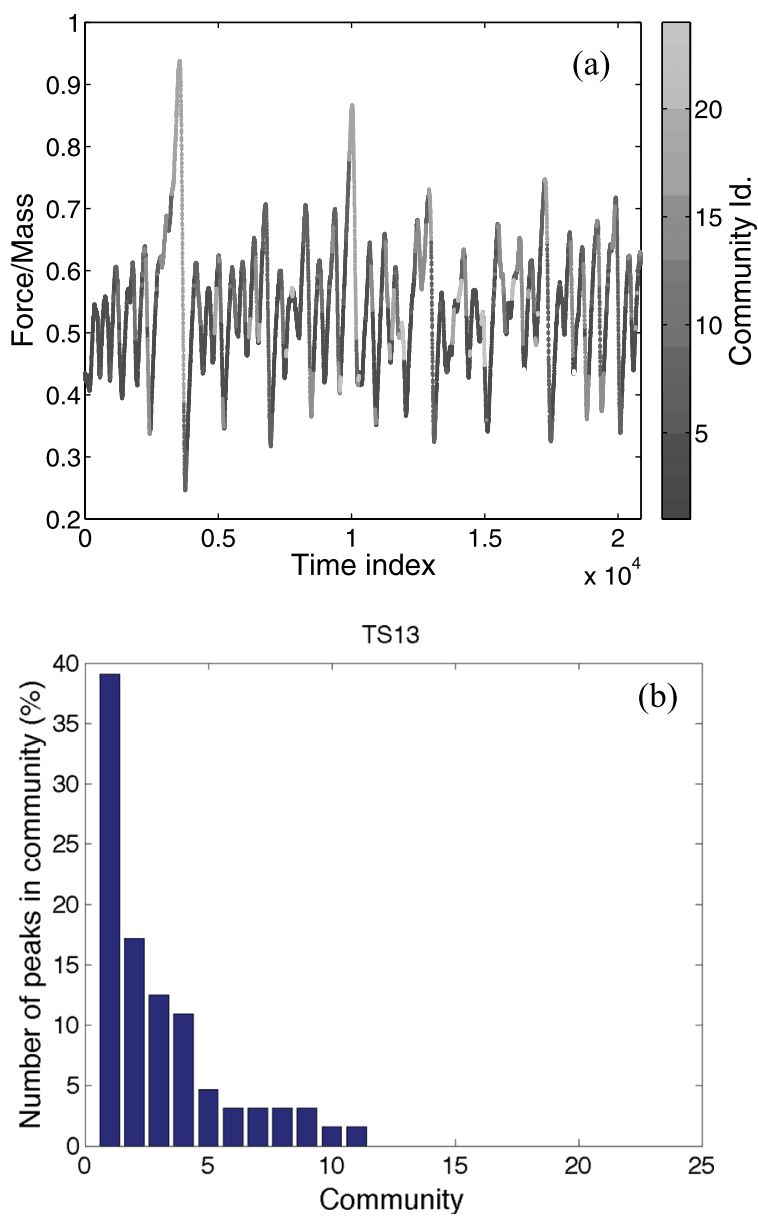

FIG. 5. TS13: (a) Community structure assignment of network nodes mapped back to the original time series. Similar color indicates membership in the same network community. (b) Community membership of time series peaks indicating onset of slip events. Communities are ordered according to the number of slip events they are associated with. 
A

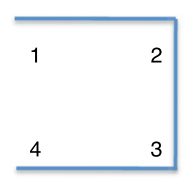

B

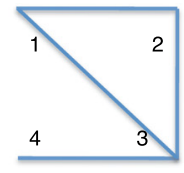

C

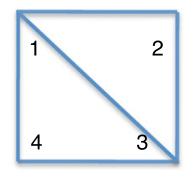

D

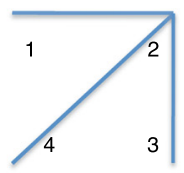

E

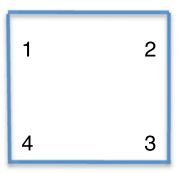

$\mathrm{F}$

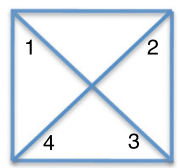

FIG. 6. The structure of the subgraphs with four connected nodes. by identifying each time series peak with the onset of a slip event, and then examine how these slip events are distributed amongst the network communities. In Fig. 5(b), we see that slip events typically lie in a small number of communities suggesting that the typical underlying dynamical behavior of slip events is the same. There are also atypical slip events that lie in their own small communities. These correspond to the larger slip events or the very small slip events seen within the time series. Network community structure identification of PSNs appears to be able to partition the dynamical behavior apparent in the time series.

\section{A. Network motifs}

The properties in Table II help to characterize the network and the underlying system at a macroscopic scale by taking global averages of local quantities. The community structure assignment provides a mesoscopic view of a network. An analysis of small subgraphs of a network is another way of studying a network at the mesoscopic scale. Subgraphs with a small number of nodes can be identified as network motifs. ${ }^{73,74}$ There are two subgraph structures consisting of three connected nodes, but only one of them is interesting, i.e., the triangle and its presence within a network is related to the clustering coefficient. The next nontrivial collection of subgraphs consists of four connected nodes. The structure and labeling of these subgraphs is shown in Fig. 6 (see Kashani et al. ${ }^{75}$ for an algorithm to identify them). The rank-ordering of their prevalence within a PSN defines superfamilies directly related to the underlying dynamics. ${ }^{54}$ In Table III, we show the rank-ordering of subgraphs with four connected nodes within PSNs when the underlying dynamical systems are: ${ }^{54}$ (i) Periodic (e.g., Rossler flow operating at parameters producing periodic orbits), (ii) Periodic +noise (e.g., a sine wave corrupted with additive Gaussian noise), (iii) Chaotic (e.g., ordinary differential equations with one positive Lyapunov exponent such as the Rossler and Lorenz flows), and (iv) Hyper-chaotic (e.g., flows with two or more positive Lyapunov exponents such as Mackey-Glass). It was also shown that these superfamilies are robust to additive Gaussian noise corruption, but as the signal-to-noise ratio goes from $30 \mathrm{~dB}$ through to $0 \mathrm{~dB}$

TABLE III. Superfamily ordering for dynamical system flows as reported in Ref. 54. The PSNs of all slider time series exhibit a new genus of superfamily ADEBCF.

\begin{tabular}{lc}
\hline \hline Flow type & Motif ranking \\
\hline Periodic & ABCFDE \\
Periodic+noise & ADBCEF \\
Chaotic & ABCDFE \\
Hyper-chaotic & ABCDEF \\
\hline \hline
\end{tabular}

the rank-ordering breaks down and becomes identical to the "Periodic+noise" superfamily. ${ }^{76}$

The difference in the rank-ordering of these subgraphs for each superfamily can be related to the character of reconstructed phase space and the trajectories therein. In sparsely populated regions of phase space, the four nearest neighbors are less likely to be mutual (i.e., non-transitive such as subgraph D) whereas points in a dense region of phase space are more likely to have mutual network connections (i.e., transitive such as subgraph F). For PSNs arising from periodic time series, subgraph $\mathrm{F}$ should appear higher compared to chaotic time series, because the trajectories should return to the same areas of phase space over and over again in periodic systems (even subject to noise). In contrast, for chaotic time series, which can be viewed as flow signposted by collections of unstable periodic orbits, there will be some return to the same area as well as some repellence from these same areas. For time series requiring a high embedding dimension to unfold their dynamics, e.g., chaotic and noise corrupted time series, the non-transitive subgraph $\mathrm{D}$ will become more frequent compared to periodic flow PSNs (cf. the superfamilies in Table III). This can also be reflected in global macroscopic averages. For example, in a PSN arising from a time series of white Gaussian noise, one observes low path length together with low clustering coefficient. Clustering coefficient is an indication of the number of triangle subgraphs in a network, here present in subgraphs B, C, and F. Thus, the low prevalence of these subgraphs in PSNs is indicative of higher dimensional dynamics or significant levels of noise corruption.

The rank-ordering of the subgraphs of four nodes for the slider PSNs exhibited the same definitive ordering, i.e., ADEBCF. That all sliders returned the same superfamily suggests the underlying (force chain) dynamics of the granular bed is consistent across the different tests. The subgraph structures which contain triangles (BCF) are the lowest ranked, consistent with the macroscopic clustering coefficient values reported in Table II. A reason for this may be attributed to the $d_{L}=4$ to 6 local dimension and higher embedding dimension of $d_{E}=8$, as well as the imposition of a large Theiler window.

The superfamily ADEBCF is also different from the classes for flows shown in Table III. Thus, in terms of motif network classification, the behavior of the slider laboratory fault appears to be a new genus of superfamily. The dynamics appear distinct from all others previously observed, and nontrivially so: i.e., high dimensional deterministic dynamics with significant noise, and no evidence of fine scale deterministic "mixing." This new genus warrants further investigation in the future.

Consider the similarities and differences of this new genus ADEBCF to the superfamily associated with noisy 
periodic dynamics, i.e., ADBCEF. The subgraph E, a square topology, is ranked higher than the subgraphs $\mathrm{BCF}$, all of which contain triangle topologies. This may be a signature of dynamical non-stationarity and not just statistical drift, i.e., one requires a long chain of connections before returning to similar states. A possible source of this subtle effect is the nature of the experiment. The dynamics of stick-slip is underpinned by the continual formation and failure by buckling of force chains in the bed. However, following each slip event, the slider moves to an undisturbed part of the bed with a new set of disks forming force chains that give rise to stick. It is possible that this leads to dynamical non-stationarity and the new superfamily. In contrast, superfamilies associated with chaos were observed in data-based models of stick-slip behavior within a biaxial compression test. ${ }^{19}$ There, however, the grains subject to deformation remain the same, if not the self-organized network of force chains they facilitate.

\section{CONCLUSION}

Inspired by past studies which consider the stick-slip of a laboratory fault as a proxy for stick-slip of a geological fault gouge, we analyzed the dynamics of stick-slip of a granular bed under shear from a slider. We complemented previous statistical analysis focussed on the distribution of the size of slip events by exploiting the concepts and techniques of complex systems theory, in particular, nonlinear time series analysis and complex networks. The number of active degrees of freedom detected from the data suggests that a real physical evolution law describing stick-slip should consider at least four to six state variables. Network community structures were able to partition the dynamical behavior apparent in the time series, which proved to be strongly associated with slip events. We also discovered that individual stick-slip events exhibited dynamics richer than a linear description and can contain a nonlinear determinism. However, models capable of exhibiting periodic dynamics are adequate for some tests. If we preserve the dynamics within a stick-slip event and effectively only shuffle the individual stick-slip cycles (ATS test), then there is insufficient evidence to reject the possibility that there are no long term dynamical correlations between the events. This makes physical sense, as stick-slip events temporally far apart in the experiment are due to force chain structures in different parts of the granular bed. That is, the bed has no long term memory. This class of data whereby we have nonlinear determinism within each stick-slip cycle, but no long term memory across stick-slip cycles, appears to also present a new stratification of systems that the network motif superfamily approach can detect.

\section{ACKNOWLEDGMENTS}

We thank the anonymous referees whose comments helped improve the manuscript. This work was supported by US ARO (W911NF-11-1-0175), the ARC (DP0986876 and DP120104759), and the Melbourne Energy Institute (AT, DMW) and HKUGC grant number PolyU 5262/11E. M.S. was supported by an ARC Future Fellowship (FT110100896) and thanks the Melbourne Energy Institute for travel support. We thank R. Xiang, X. Xu, and Y. Chang for discussions during the preparation of this manuscript.

${ }^{1}$ D. W. Lee, X. Banquy, and J. N. Israelachvili, Proc. Natl. Acad. Sci. U.S.A. 110, E567 (2013).

${ }^{2}$ U. Galvanetto, S. Bishop, and L. Briseghella, Int. J. Bifurcation Chaos Appl. Sci. Eng. 5, 637 (1995).

${ }^{3}$ K. Popp and P. Stelter, Philos. Trans. R. Soc. London, Ser. A 332, 89 (1990).

${ }^{4}$ D. Karnopp, J. Dyn. Syst.-Trans. ASME 107, 100 (1985).

${ }^{5}$ C. Collettini, A. Niemeijer, C. Viti, and C. Marone, Nature 462, 907 (2009).

${ }^{6}$ K. Christensen, L. Danon, T. Scanlon, and P. Bak, Proc. Natl. Acad. Sci. U.S.A. 99, 2509 (2002).

${ }^{7}$ E. Aharonov and D. Sparks, J. Geophys. Res. 109, B09306, doi:10.1029/2003JB002597 (2004).

${ }^{8}$ M. L. Gee, P. M. McGuiggan, J. Israelachvili, and A. M. Homola, J. Chem. Phys. 93, 1895 (1990).

${ }^{9}$ J. Geng and R. P. Behringer, Phys. Rev. E 71, 011302 (2005).

${ }^{10}$ Y. Guo and J. K. Morgan, J. Geophys. Res. 109, B12305, doi:10.1029/2004JB003044 (2004).

${ }^{11}$ I. Albert, P. Tegzes, R. Albert, A.-L. Barabási, T. Vicsek, B. Kahng, and P. Schiffer, Phys. Rev. E 64, 031307 (2001).

${ }^{12}$ I. Albert, B. Kahng, R. Albert, J. G. Sample, M. Pfeifer, A.-L. Barabási, T. Vicsek, and P. Schiffer, Phys. Rev. Lett. 84, 5122 (2000).

${ }^{13}$ R. Albert, M. A. Pfeifer, A.-L. Barabási, and P. Schiffer, Phys. Rev. Lett. 82, 205 (1999).

${ }^{14}$ K. A. Alshibli and L. E. Roussel, Int. J. Numer. Anal. Met. 30, 1391 (2006).

${ }^{15}$ F. B. Bowden and D. Tabor, The Friction and Lubrication of Solids (Oxford University Press, New York, USA, 1950).

${ }^{16}$ P. A. Johnson, H. Savage, M. Knuth, J. Gomberg, and C. Marone, Nature 451, 57 (2008).

${ }^{17}$ M. Herrera, S. McCarthy, S. Slotterback, E. Cephas, W. Losert, and M. Girvan, Phys. Rev. E 83, 061303 (2011).

${ }^{18}$ E. Kolb, T. Mazoz, E. Clement, and J. Duran, Eur. Phys. J. B 8, 483 (1999).

${ }^{19}$ M. Small, D. M. Walker, A. Tordesillas, and C. K. Tse, Chaos 23, 013113 (2013).

${ }^{20}$ G. Chambon, J. Schmittbuhl, A. Corfdir, J. P. Vilotte, and S. Roux, Phys. Rev. E 68, 011304 (2003).

${ }^{21}$ F. Peer and T. Venter, J. South Afr. Inst. Min. Metall. 103, 403 (2003).

${ }^{22}$ M. Knuth and C. Marone, Geochem., Geophys., Geosyst. 8, Q03012, doi:10.1029/2006GC001327 (2007).

${ }^{23}$ K. Mair, K. M. Frye, and C. Marone, J. Geophys. Res. 107, 2219, doi:10.1029/2001JB000516 (2002).

${ }^{24}$ K. E. Daniels and N. W. Hayman, J. Geophys. Res. 113, B11411, doi:10.1029/2008JB005781 (2008).

${ }^{25}$ N. W. Hayman, L. Ducloué, K. L. Foco, and K. E. Daniels, Pure Appl. Geophys. 168, 2239 (2011).

${ }^{26}$ F. Radjai, D. E. Wolf, M. Jean, and J.-J. Moreau, Phys. Rev. Lett. 80, 61 (1998).

${ }^{27}$ T. S. Majmudar and R. P. Behringer, Nature 435, 1079 (2005).

${ }^{28}$ D. Howell, R. P. Behringer, and C. Veje, Phys. Rev. Lett. 82, 5241 (1999).

${ }^{29}$ T. S. Majmudar, M. Sperl, S. Luding, and R. P. Behringer, Phys. Rev. Lett. 98, 058001 (2007).

${ }^{30}$ D. Bi, J. Zhang, B. Chakraborty, and R. P. Behringer, Nature 480, 355 (2011).

${ }^{31}$ J. Zhang, T. Majmudar, A. Tordesillas, and R. P. Behringer, Granular Matter 12, 159 (2010)

${ }^{32}$ A. Tordesillas, D. M. Walker, F. G. J. Zhang, and R. P. Behringer, Phys. Rev. E 86, 011306 (2012).

${ }^{33}$ A. Tordesillas, Q. Lin, J. Zhang, R. P. Behringer, and J. Shi, J. Mech. Phys. Solids 59, 265 (2011).

${ }^{34}$ A. Tordesillas, Philos. Mag. 87, 4987 (2007).

${ }^{35}$ D. M. Walker and A. Tordesillas, Int. J. Solids Struct. 47, 624 (2010).

${ }^{36}$ A. Tordesillas, D. M. Walker, and Q. Lin, Phys. Rev. E 81, 011302 (2010).

${ }^{37}$ D. M. Walker and A. Tordesillas, Phys. Rev. E 85, 011304 (2012).

${ }^{38}$ L. Kondic, A. Goullet, C. S. O'Hern, M. Kramar, K. Mischaikow, and R. P. Behringer, Europhys. Lett. 97, 54001 (2012). 
${ }^{39}$ M. Kramar, A. Goullet, L. Kondic, and K. Mischaikow, Phys. Rev. E 87, 042207 (2013).

${ }^{40}$ J. Krim, P. Yu, and R. P. Behringer, Pure Appl. Geophys. 168, 2259 (2011).

${ }^{41}$ H. Kantz and T. Schreiber, Nonlinear Time Series Analysis (Cambridge University Press, Cambridge, 1997).

${ }^{42}$ M. Small, Applied Nonlinear Time Series Analysis: Applications in Physics, Physiology and Finance, Vol. 52 Nonlinear Science Series A (World Scientific, Singapore, 2005).

${ }^{43}$ M. Small and C. K. Tse, IEEE Trans. Circuits-I 50, 663 (2003).

${ }^{44}$ A. M. Fraser and H. L. Swinney, Phys. Rev. A 33, 1134 (1986).

${ }^{45}$ H. D. I. Abarbanel, Analysis of Observed Chaotic Data (Springer-Verlag, New York, 1996).

${ }^{46}$ T. Nakamura, M. Small, and Y. Hirata, Phys. Rev. E 74, 026205 (2006).

${ }^{47} \mathrm{~F}$. Takens, in Lecture Notes in Mathematics (Springer-Verlag, Berlin, 1981), Vol. 898, p. 366.

${ }^{48}$ H. D. I. Abarbanel and M. B. Kennel, Phys. Rev. E 47, 3057 (1993).

${ }^{49}$ M. Small, D. Yu, and R. G. Harrison, Phys. Rev. Lett. 87, 188101 (2001).

${ }^{50}$ J. Theiler and P. E. Rapp, Electron Clin. Neuro. 98, 213 (1996).

${ }^{51}$ A. Tordesillas, J. Shi, and J. F. Peters, Granular Matter 14, 295 (2012).

${ }^{52}$ J. Zhang and M. Small, Phys. Rev. Lett. 96, 238701 (2006).

${ }^{53}$ L. Lacasa, B. Luque, F. Ballesteros, J. Luque, and J. C. Nuno, Proc. Natl. Acad. Sci. U.S.A. 105, 4972 (2008).

${ }^{54}$ X. Xu, J. Zhang, and M. Small, Proc. Natl. Acad. Sci. U.S.A. 105, 19601 (2008).

${ }^{55}$ N. Marwan, J. F. Donges, Y. Zou, R. V. Donner, and J. Kurths, Phys. Lett. A 373, 4246 (2009).

${ }^{56}$ R. V. Donner, M. Small, J. F. Donges, N. Marwan, Y. Zou, R. Xiang, and J. Kurths, Int. J. Bifurcation Chaos Appl. Sci. Eng. 21, 1019 (2011).

${ }^{57}$ J. B. Tenenbaum, V. de Silva, and J. C. Langford, Science 290, 2319 (2000).
${ }^{58}$ E. Bollt, Int. J. Bifurcation Chaos Appl. Sci. Eng. 17, 1199 (2007).

${ }^{59}$ H. Suetani, K. Soejima, R. Matsuoka, U. Parlitz, and H. Hata, Phys. Rev. E 86, 036209 (2012).

${ }^{60}$ G. Froyland, Discret. Contin. Dyn. Syst. 17(3), 203 (2007).

${ }^{61}$ M. Dellnitz, G. Froyland, and S. Sertl, Nonlinearity 13, 1171 (2000).

${ }^{62}$ G. Froyland and M. Dellnitz, SIAM J. Sci. Comput. 24, 1839 (2003).

${ }^{63}$ G. Froyland, Physica D 200, 205 (2005).

${ }^{64}$ E. M. Bollt, L. Billings, and I. B. Schwartz, Physica D 173, 153 (2002).

${ }^{65}$ E. M. Bollt, A. Luttman, S. Kramer, and R. Basnayake, Int. J. Bifurcation Chaos Appl. Sci. Eng. 22, 1230012 (2012).

${ }^{66}$ N. Santitissadeekorn and E. M. Bollt, Physica D 231, 95 (2007).

${ }^{67}$ Y. Hirata, K. Judd, and D. Kilminster, Phys. Rev. E 70, 016215 (2004).

${ }^{68} \mathrm{D}$. Lind and B. Marcus, An Introduction to Symbolic Dynamics and Coding (Cambridge University Press, Cambridge, 1995).

${ }^{69}$ B. P. Kitchens, Symbolic Dynamics: One-sided, Two-sided and Countable State Markov Shifts (Springer-Verlag, Berlin, 1998).

${ }^{70}$ M. E. J. Newman, Networks: An Introduction (Oxford University Press, Oxford, 2010).

${ }^{71}$ Wolfram-Research Mathematica, Version 7.0 (Wolfram Research, Inc. Champaign, IL, 2008).

${ }^{72}$ A. Clauset, Phys. Rev. E 72, 026132 (2005).

${ }^{73}$ R. Milo, S. Shen-Orr, S. Itzkovitz, N. Kashtan, D. Chklovskii, and U. Alon, Science 298, 824 (2002).

${ }^{74}$ R. Milo, S. Itzkovitz, N. Kashtan, R. Levitt, S. Shen-Orr, I. Ayzenshtat, M. Sheffer, and U. Alon, Science 303, 1538 (2004).

${ }^{75}$ Z. R. M. Kashani, H. Ahrabian, E. Elahi, A. Nowzari-Dalini, E. S. Ansari, S. Asadi, S. Mohammadi, F. Schreiber, and A. Masoudi-Nejad, BMC Bioinform. 10, 318 (2009).

${ }^{76}$ R. Xiang, J. Zhang, X.-K. Xu, and M. Small, Chaos 22, 013107 (2012). 